\title{
ON THE SECTIONAL CURVATURE OF COMPACT HYPERSURFACES
}

\author{
LESLIE COGHLAN AND YOE ITOKAWA
}

(Communicated by Jonathan M. Rosenberg)

\begin{abstract}
We establish a sufficient condition for compact hypersurfaces of a complete riemannian manifold to be spherical. It is well known, from the works of Jacobowitz, Jorge and Koutroufiotis, and others, that the maximum sectional curvature of such hypersurfaces can be estimated from the curvature of the ambient space and the outer radius. Our result sharpens these estimates. It also implies a new nonimmersibility theorem of the Chern-Kuiper type.
\end{abstract}

\section{INTRODUCTION}

Let $M$ be a compact riemannian manifold of dimension $d$ and of class $C^{\infty}$. Chern and Kuiper [6] showed that if the sectional curvature of $M$ is everywhere nonpositive, then $M$ cannot be immersed isometrically into $\mathbf{R}^{d+k}$ for any $k \leq d-1$. Jacobowitz proved that if $f: M \rightarrow \mathbf{R}^{d+k}, k \leq d-1$, is an isometric immersion and $f(M)$ is contained in a metric ball of radius $\rho$, then the sectional curvature of $M$ satisfies $\sup K \geq 1 / \rho^{2}$. In [3] and [4], Beltagy proved analogous results for immersions $f: M \rightarrow M^{d+k}(c)$ where $M^{d+k}(c)$ is the simply connected space form of curvature $c \neq 0$ and dimension $d+k$, with $k \leq d-1$ once again. Namely, he found that if $c<0$, then $\sup K \geq$ $-c \operatorname{csch}^{2}(\sqrt{-c} \rho)$, and if $c>0$ and $\rho \leq \pi / 2(\sqrt{c})$, then $\sup K \geq c \csc ^{2}(\sqrt{c} \rho)$. The purpose of the present paper is to describe for the case of hypersurfaces what happens in the event that the inequality is attained in these results. More precisely, we prove the following theorem.

Theorem 1. Let $f: M \rightarrow M^{d+1}(c)$ be an isometric immersion of a compact riemannian manifold of dimension $d \geq 2$ into a simply connected space form. Suppose that $f(M)$ is contained in a metric ball of radius $\rho$. Assume either one of the following conditions:

- $c<0$ and $\sup K=-c \operatorname{csch}^{2}(\sqrt{-c} \rho)$

- $c=0$ and $\sup K=1 / \rho^{2}$,

- $c>0, \rho \leq \pi /(2 \sqrt{c})$, and $\sup K=c \csc ^{2}(\sqrt{c} \rho)$

Then $f(M)$ is the boundary of the metric ball.

Received by the editors August 13, 1989.

1980 Mathematics Subject Classification (1985 Revision). Primary 53C40; Secondary 53C 42.

The authors were supported in part by NSF EPSCoR grant No. RII-8610669.

(C) 1990 American Mathematical Society $0002-9939 / 90 \$ 1.00+\$ .25$ per page 
Similar rigidity phenomena were previously observed for the estimate of the mean curvature by Markvorsen [11], and, for the case $d=2$, Koutroufiotis [10] proved Theorem 1. Actually, the proof in [10] is only for the case $c=0$ but a closer examination of the proof shows that the argument is valid for arbitrary $c$. It does not generalize to higher dimensions, however. For $d \geq 3$, Theorem 1 is a special case of the following theorem.

Theorem 2. Suppose that $M$ is a compact manifold of dimension $d \geq 3$ and let $f: M \rightarrow \bar{M}$ be an isometric immersion into a complete manifold of dimension $d+1$. Suppose that $f(M)$ is contained in some closed normal metric ball $B_{\rho}(o)^{-}$ in $\bar{M}$ of radius $\rho$. Let $c$ be an upper bound of the sectional curvature $\bar{K}$ of $\bar{M}$ in $B_{\rho}(o)$. If $c>0$, assume that $\rho \leq \pi /(2 \sqrt{c})$. Then there exists a positive real number $\mu_{c}(\rho)$ depending only on $c$ and $\rho$, so that if the sectional curvature of $M$ satisfies the condition

$$
K(\sigma) \leq \bar{K}\left(f_{*}(\sigma)\right)+\mu_{c}(\rho)^{2} / \rho^{2}
$$

for all 2 planes $\sigma$ tangent to $M$, then $B_{\rho}(o)^{-}$is isometric to a metric ball of radius $\rho$ in $M^{d+1}(c)$ and $M$ coincides with its boundary.

The actual expression for $\mu_{c}(\rho)$ will be given in $\S 2$ as formula (2.1). Just as Theorem 1 sharpens the theorems of Jacobowitz and Beltagy in the special case of codimension 1, Theorem 2 sharpens a result of Jorge and Koutroufiotis [8] and Baikousis and Brickell [1] in a special case. They prove for a complete submanifold $M$ of dimension $d$ and codimension $\leq d-1$, with bounded scalar curvature contained in a closed ball of radius $\rho$, that $\sup K \geq \inf K+$ $\mu_{c}(\rho)^{2} / \rho^{2}$. The definition of the function $\mu_{c}$ was taken from [8].

Besides Theorem 1, Theorem 2 has as immediate consequences the following nonimmersibility theorems of Chern-Kuiper type.

Corollary 1. Let $\bar{M}$ be a simply connected $d+1$ dimensional manifold, $d \geq 3$, whose sectional curvature is pinched,

$$
b \leq \bar{K} \leq c,
$$

where $b$ and $c$ are constants. Suppose that $o \in \bar{M}$ is a point such that the sectional curvature at $o$ is variable and $B_{\rho}(o)^{-}$is a closed normal ball. If $c>0$, assume also that $\rho<\pi /(2 \sqrt{c})$. Let $M$ be a compact $d$ dimensional manifold whose curvature satisfies

$$
K \leq b+\mu_{c}(\rho)^{2} / \rho^{2}
$$

Then $M$ cannot be immersed isometrically in $B_{\rho}(o)^{-}$.

Also, since the boundary of a normal ball in a space form is isometric to a sphere of constant curvature, we obtain the following corollary.

Corollary 2. Let $\bar{M}$ be a manifold as in Corollary 1 . Let $M$ be a compact $d$ dimensional manifold which satisfies

$$
K \leq b+\mu_{c}(\rho)^{2} / \rho^{2}
$$


Then $M$ cannot be immersed isometrically in any closed normal ball of radius $\leq \rho$ in $\bar{M}$ unless $M$ is isometric to a $d$ dimensional sphere of constant sectional curvature.

In this paper, we will review some definitions and preliminary calculations in $\S 2$. Then, in $\S 3$, we will prove Theorem 2 . The key step in $\S 3$ is Lemma 3.1 , where we prove the subharmonicity of a certain modified distance function from $o$. Theorem 1 follows from Theorem 2 as an easy corollary once $\mu_{c}$ is explicitly defined.

\section{Preliminaries}

Let $M$ be a compact riemannian manifold of dimension $d \geq 3$ and $\bar{M}$ a complete manifold of dimension $d+1$. Let $f: M \rightarrow \bar{M}$ be an isometric immersion. We assume the manifolds and the immersion to be differentiable of class $C^{\infty}$ for the sake of simplicity, although all of the arguments hold for class $C^{r}$ for sufficiently large $r$.

We denote by $\alpha \in \Gamma\left(T^{*} M \otimes T^{*} M \otimes M\right)$ the second fundamental form of $f$. Fix a point $o \in \bar{M}$, and let $d_{o}(p)$ denote the distance from any point $p \in \bar{M}$ to $o$. Set $\bar{\varphi}:=\frac{1}{2}\left(d_{o}\right)^{2}$ and by $\mathbf{g}$ the gradient vector field of $\bar{\varphi}$ in $\bar{M}$. At any $p,\left|\mathbf{g}_{p}\right|=d_{o}(p)$. Define a function $\varphi: M \rightarrow \mathbf{R}$ by $\varphi(x):=\bar{\varphi}(f(x))$. Note that $\varphi$ is differentiable if $f(M)$ is contained inside a normal ball.

Let $c$ be a real number. We define a function $\mu_{c}$ where $\mu_{c}: \mathbf{R}_{+} \rightarrow \mathbf{R}_{+}$in the case $c \leq 0$ and $\mu_{c}:[0, \pi /(2 \sqrt{c})) \rightarrow \mathbf{R}_{+}$for the case $c>0$ by

$$
\mu_{c}(t):= \begin{cases}t \sqrt{-c} \operatorname{coth}(t \sqrt{-c}), & c<0 \\ 1, & c=0 \\ t \sqrt{c} \cot (t \sqrt{c}), & c>0 .\end{cases}
$$

Suppose that $\bar{M}=M^{d+1}(c)$ is the simply connected space form of curvature $c$. If $c>0$, assume also that $f(M)$ is contained in the ball $B_{\pi /(2 \sqrt{c})}(o)^{-}$. In this case, the Hessian of the function $\varphi$ at a point $x \in M$ is given by

$$
\text { hess }_{x} \varphi(\mathbf{v}, \mathbf{w})=\mu_{c}\left[d_{o}(f(x))\right]\langle\mathbf{v}, \mathbf{w}\rangle+\left\langle\mathbf{g}_{f(x)}, \alpha(\mathbf{v}, \mathbf{w})\right\rangle,
$$

for $\mathbf{v}, \mathbf{w} \in T_{\underline{x}} M$. If, on the other hand, $\bar{M}$ has variable curvature, let $o$ be any point in $\bar{M}$ and assume that $f(M)$ is contained wholly in some normal neighborhood of $o$. Choose a number $c \geq \sup \bar{K}(\sigma)$, where $\bar{K}$ is the sectional curvature of $\bar{M}$ and $\sigma$ ranges over all two-dimensional planes that are tangent to the minimal geodesics joining $o$ with points in $f(M)$. If $c>0$, we also assume that $\sup _{x \in M} d_{o}(f(x)) \leq \pi /(2 \sqrt{c})$. In this case, we have

$$
\text { hess }_{x} \varphi(\mathbf{v}, \mathbf{v}) \geq \mu_{c}\left[d_{o}(f(x))\right]|\mathbf{v}|^{2}+\left\langle\mathbf{g}_{f(x)}, \alpha(\mathbf{v}, \mathbf{v})\right\rangle
$$

for all $\mathbf{v} \in T_{x} M$. Moreover, equality is obtained only if $\bar{K}(\tau) \equiv c$ for all 2 planes $\tau$ spanned by $\dot{\gamma}$ and $\mathbf{V}$ where $\gamma:[0,1] \rightarrow M$ is a minimal geodesic connection from $o$ to $f(x)$ and $\mathbf{V}$ is the Jacobi field along $\gamma$ with the boundary value condition $\mathbf{V}(0)=\mathbf{O}$ and $\mathbf{V}(1)=f_{*}(\mathbf{v})$. For the proof, see [8]. 
(2.4) Lemma. Suppose that $f(M) \subset B_{\rho}(o)^{-}$. Let $p \in M$ be a local maximum point for the function $\varphi$. Then for any 2 plane $\sigma \subset T_{p} M$, the sectional curvature $K$ of $M$ and $\bar{K}$ of $\bar{M}$ are related by

$$
K(\sigma) \geq \bar{K}\left(f_{*}(\sigma)\right)+\mu_{c}(\rho)^{2} / \rho^{2} .
$$

In [8], an inequality is proven concerning the supremum of the left side under a weaker assumption; namely, $M$ is assumed only to be complete with scalar curvature bounded below and the codimension of $M$ in $\bar{M}$ is assumed to be less than $d$. Our proof is essentially identical to that case.

Proof. Since $p$ is a maximum point for $\varphi$, for any $\mathbf{v} \in T_{p} M$, hess $p(\mathbf{v}, \mathbf{v}) \leq$ 0 . Now let $\kappa_{1}, \ldots, \kappa_{d}$ be the principal curvatures of the immersion at $p$, and let $\mathbf{e}_{1}, \ldots, \mathbf{e}_{d} \in T_{p} M$ be the unit vectors in the corresponding principal directions. Then, for each $i$, by (2.3) and the Cauchy-Schwartz inequality,

$$
\begin{aligned}
0 & \geq \operatorname{hess}_{p} \varphi\left(\mathbf{e}_{i}, \mathbf{e}_{i}\right) \geq \mu_{c}\left[d_{o}(f(p))\right]+\left\langle\mathbf{g}_{f(p)}, \alpha\left(\mathbf{e}_{i}, \mathbf{e}_{i}\right)\right\rangle \\
& \geq \mu_{c}\left[d_{o}(f(p))\right]-\left|\mathbf{g}_{f(p)}\right|\left|\alpha\left(\mathbf{e}_{i}, \mathbf{e}_{i}\right)\right| \\
& =\mu_{c}\left[d_{o}(f(p))\right]-d_{o}(f(p)) \kappa_{i} .
\end{aligned}
$$

Rearranging the equation, we have

$$
\kappa_{i} \geq \mu_{c}\left[d_{o}(f(p))\right] / d_{o}(f(p)) .
$$

Now, noting that $\mu_{c}(t) / t$ is a monotone decreasing function on $t>0$ for $c \leq 0$ and on $[0, \pi /(2 \sqrt{c}))$ for $c>0$, and that $d_{o}(f(p)) \leq \rho$ by assumption, we have

$$
\kappa_{i} \geq \mu_{c}(\rho) / \rho
$$

for all $i=1, \ldots, d$.

Now choose an orthonormal basis $\{\mathbf{v}, \mathbf{w}\}$ of the plane $\sigma$. Set $\mathbf{v}=\sum v^{i} \mathbf{e}_{i}$ and $\mathbf{w}=\sum w^{i} \mathbf{e}_{i}$. Then the Gauss equation is

$$
K(\sigma)=\bar{K}\left(f_{*}(\sigma)\right)+\sum_{i<j}\left(v^{i} w^{j}-w^{i} v^{j}\right)^{2} \kappa_{i} \kappa_{j} .
$$

Substituting in (2.5),

$$
\begin{aligned}
K(\sigma) & \geq \bar{K}\left(f_{*}(\sigma)\right)+\sum_{i<j}\left(v^{i} w^{j}-w^{i} v^{j}\right)^{2} \cdot\left[\mu_{c}(\rho) / \rho\right]^{2} \\
& =\bar{K}\left(f_{*}(p)\right)+\left[\mu_{c}(\rho) / \rho\right]^{2}|\mathbf{v} \wedge \mathbf{w}|^{2} .
\end{aligned}
$$

Since $\mathbf{v}$ and $\mathbf{w}$ were chosen orthonormal, we obtain the desired inequality.

\section{Proof of Theorem 2}

Fix a point $o \in \bar{M}$. First, assume that $M$ is connected. Also assume that $f(M) \subset B_{\rho}(o)^{-}$where $B_{\rho}(o)$ is a normal ball. Set $c:=\sup \bar{K}(\bar{\sigma})$ where $\bar{\sigma}$ ranges over all tangent 2-plane sections over $B_{p}(0)$. Assume that for all 2 planes $\sigma$ tangent to $M, K(\sigma) \leq \bar{K}\left(f_{*}(\sigma)\right)+\mu_{c}(\rho)^{2} / \rho^{2}$. First, we claim that 
there is a point $p \in M$ so that $d_{0}(f(p))=\rho$. Otherwise, for some $\rho^{\prime}<\rho$, $f(M) \subset B_{\rho^{\prime}}(o)^{-}$, and by Lemma 2.4 , there would be a point $q \in M$ so that for any $\sigma \subset T_{q} M, K(\sigma) \geq \bar{K}\left(f_{*}(\sigma)\right)+\mu_{c}(\rho)^{2} / \rho^{2}>\bar{K}\left(f_{*}(\sigma)\right)+\mu_{c}(\rho)^{2} / \rho^{2}$. Clearly, such a point $p$ is a maximum point for $\varphi$.

3.1. Lemma. Let $p$ be defined as previously noted. Then there is a neighborhood $U$ of $p$ in $M$ so that $\varphi \mid U$ is subharmonic.

Proof. Let $\kappa_{1}, \ldots, \kappa_{d}$ be the principal curvature functions of $f$. For each $x \in M$, let $\mathbf{e}_{1, x}, \ldots, \mathbf{e}_{d x} \in T_{x} M$, be the unit vectors in the corresponding principal directions. Denote by $\sigma_{i j x}$ the plane in $T_{x} M$ spanned by $\mathbf{e}_{i x}$ and $\mathbf{e}_{j x}$.

Then, since $p$ is a maximum point for $\varphi$, by $(2.5), \kappa_{i}(p) \geq \mu_{c}(\rho) / \rho>0$ for each $i$. On the other hand, by (2.6),

$$
\bar{K}\left(f_{*}\left(\sigma_{i j p}\right)+\mu_{c}(\rho)^{2} / \rho^{2} \geq K\left(\sigma_{i j p}\right)=\bar{K}\left(f_{*}\left(\sigma_{i j p}\right)\right)+\kappa_{i}(p) \kappa_{j}(p)\right.
$$

so each $\kappa_{i}=\mu_{c}(\rho) / \rho$ precisely, i.e., $p$ is an umbilic point of $f$. Let $U$ be a neighborhood of $p$ in $M$ so that for all $x \in U$ and for all $i=1, \ldots, d$, $\kappa_{i}(x)>0$ and

$$
\left|\kappa_{i}(x)-\mu_{c}(\rho) / \rho\right|<(d-2) \mu_{c}(\rho) / \rho .
$$

Note that here we are using the assumption that $d \geq 3$. We claim that on $U$, $\Delta \varphi \geq 0$.

By way of contradiction, assume that $\Delta \varphi(q)<0$ at some $q \in U$. Then, taking the trace of (2.3),

$$
0>\Delta \varphi(q) \geq d \cdot \mu_{c}(\rho)+\left\langle\mathbf{g}_{f(q)}, d \cdot \mathbf{H}_{q}\right\rangle,
$$

where $\mathbf{H}$ is the mean curvature vector of $f$. By the Cauchy-Schwartz inequality,

$$
0>d \cdot \mu_{c}(\rho)-\left|\mathbf{g}_{f(q)}\right||d \mathbf{H}| \geq d \cdot \mu_{c}(\rho)-\rho\left(\kappa_{1}(q)+\ldots+\kappa_{d}(q)\right),
$$

or

$$
\kappa_{1}(q)+\cdots+\kappa_{d}(q)>d\left(\mu_{c}(\rho) / \rho\right) .
$$

For convenience, we may assume that $\kappa_{1}, \ldots, \kappa_{d}$ are arranged in the descending order $\kappa_{1}(q) \geq \kappa_{2}(q) \geq \ldots \geq \kappa_{d}(q)$. If $\kappa_{1}(q)$ and $\kappa_{2}(q)$ are both $>\mu_{c}(\rho) / \rho$, then $K\left(\sigma_{12 q}\right)>\bar{K}\left(f_{*}\left(\sigma_{12 q}\right)\right)+\mu_{c}(\rho)^{2} / \rho^{2}$, contradicting our curvature assumption. Assume that $\kappa_{1}(q)>\mu_{c}(\rho) / \rho$, but $\kappa_{2}(q) \leq \mu_{c}(\rho) / \rho$.

Set $\varepsilon:=\kappa_{1}(q)-\mu_{c}(\rho) / \rho$. Then

$$
\mu_{c}(\rho) / \rho+\varepsilon+(d-1) \kappa_{2}(q) \geq \kappa_{1}(q)+\ldots+\kappa_{d}(q)>d\left(\mu_{c}(\rho) / \rho\right),
$$

so

$$
\kappa_{2}(q)>\mu_{c}(\rho) / \rho-\varepsilon /(d-1) .
$$


Hence,

$$
\begin{aligned}
K\left(\sigma_{12 q}\right)= & \bar{K}\left(f_{*}\left(\sigma_{12 q}\right)\right)+\kappa_{1}(q) \kappa_{2}(q) \\
> & \bar{K}\left(f_{*}\left(\sigma_{12 q}\right)\right)+\left(\mu_{c}(\rho) / \rho+\varepsilon\right)\left[\mu_{c}(\rho) / \rho-\varepsilon /(d-1)\right] \\
= & \bar{K}\left(f_{*}\left(\sigma_{12 q}\right)\right)+\mu_{c}(\rho)^{2} / \rho^{2}+\left(\frac{\mu_{c}(\rho)}{\rho}-\frac{\mu_{c}(\rho)}{(d-1) \rho}\right) \varepsilon-\frac{\varepsilon^{2}}{d-1} \\
= & \bar{K}\left(f_{*}\left(\sigma_{12 q}\right)\right)+\mu_{c}(\rho)^{2} / \rho^{2} \\
& \quad+\frac{\varepsilon}{d-1}\left[(d-2) \mu_{c}(\rho) / \rho-\varepsilon\right] .
\end{aligned}
$$

Since $0<\varepsilon<(d-2) \mu_{c}(\rho) / \rho$, this again contradicts our curvature assumption. Hence, $\Delta \varphi(x) \geq 0$ for all $x \in U$.

Lemma 3.1 implies by the maximum principle for subharmonic functions that $\varphi(x) \equiv \varphi(p)=\frac{1}{2} \rho^{2}$ on $U$. But this implies that whenever $p \in M$ has $d_{0}(f(p))=\rho$, then $d_{0} \circ f \equiv \rho$ in a neighborhood of $p$. Since we are assuming that $M$ is connected, $d_{0}(f(x))=\rho$ for all $x \in M$ and $f(M)$ coincides with $\partial B_{\rho}(o)$.

Moreover, the inequalities in (2.3) and (2.4) must be equalities at each point in $M$. By the equality given by (2.3), the sectional curvatures along every geodesic joining $o$ with a point of $f(M)$ must equal $c$ identically. It is then a standard argument to show that $B_{\rho}(o)^{-}$is isometric to a ball in $M^{d+1}(c)$. For instance, since $B_{\rho}(0)^{-}$is assumed to be normal, one may use the Rauch Comparison theorem. See, for example, the book [5].

Lastly, suppose that $M$ is not connected. Then the $f$ image of each connected component of $M$ coincides with $\partial B_{\rho}(o)$. However, since $B_{\rho}(o)$ is a normal ball, $\partial B_{\rho}(o)$ is connected. This completes the proof of Theorem 2.

\section{REFERENCES}

1. C. Baikousis and F. Brickell, A note on isometric immersions, J. Austral. Math. Soc. Ser. A 33 (1982), 162-166.

2. C. Baikousis and T. Koufogiorgos, Isometric immersions of complete Riemannian manifolds into euclidean space, Proc. Amer. Math. Soc. 79 (1980), 87-88.

3. M. A. Beltagy, Isometric embedding of a compact Riemannian manifold into a sphere, Indian J. Pure Appl. Math. 14 (1983), 1406-1411.

4. M. A. Beltagy, Isometric embedding of a compact Riemannian manifold into hyperbolic space, J. Indian Math. Soc. (N.S.) 48 (1984), 81-87.

5. J. Cheeger and D. Ebin, Comparison theorems in Riemannian Geometry, North-Holland, Amsterdam and Oxford, 1975.

6. S. S. Chern and N. H. Kuiper, Some theorems on the isometric embedding of compact Riemannian manifolds in euclidean space, Ann. of Math. 56 (1952), 442-430.

7. H. Jacobowitz, Isometric embedding of a compact Riemannian manifold into euclidean space, Proc. Amer. Math. Soc. 40 (1973), 245-246.

8. L. Jorge and D. Koutroufiotis, An estimate for the curvature of bounded submanifolds, Amer. J. Math. 103 (1981), 711-725. 
9. L. Jorge and F. Xavier, An inequality between the exterior diameter and the mean curvature of bounded immersion, Math. Z. 178 (1981), 78-82.

10. D. Koutroufiotis, Elementary geometric applications of a maximum principle for nonlinear elliptic operators, Arch. Math. 24 (1973), 97-99.

11. S. Markvorsen, $A$ sufficient condition for a compact immersion to be spherical, Math. Z. 183 (1983), 407-411.

Department of Mathematics, University of Alabama at Birmingham, Birmingham, AlABAMA 35294 